\title{
LOCATION-BASED MANAGEMENT OF CONSTRUCTION PROJECTS: PART OF A NEW TYPOLOGY FOR PROJECT SCHEDULING METHODOLOGIES
}

\author{
Russell Kenley \\ Business and Enterprise \\ Swinburne University of Technology \\ AUSTRALIA
}

\author{
Olli Seppänen \\ Vico Software \\ 1966 13th Street, Suite 250 \\ Boulder, CO 80302 USA
}

\begin{abstract}
The domain of project scheduling has been treated as various disparate techniques, each competing to be the best method for effective planning and control of projects. Especially, there are many terms used to describe the family of construction scheduling methods which variously involve repetition, linear relationships, vertical or horizontal staging or in some other way map production units against time. This paper considers the underlying rationale behind those terms and proposes a new typology and terminology to encapsulate the entire family of scheduling methods and specifically to differentiate the family of production-oriented methodologies from the more common critical path methodologies. For this purpose the following terms are proposed: activity-based methodologies which may be divided into deterministic and probabilistic, and location-based methodologies which may be divided into unit production and location production. The paper then shows how location production in the location-based methodology involves focusing on locations as the unit of analysis and tasks as the unit of control as the framework for production management.
\end{abstract}

\section{INTRODUCTION}

Planning and scheduling construction has been an active topic of research since the late 1950s and in that time a plethora of techniques and methods has arisen. In that time, industry as adopted the critical path method as the dominant paradigm for practical scheduling. Yet, in the research community, there has been a stubborn reluctance to accept this final answer and considerable activity over a lengthy period of time has kept alive an alternative family of methods. While it may be tempting to brand these as academic playthings, the reality is that industry has much to gain from taking a more inclusive approach. There is much to be gained from drawing from the strengths of all techniques, but this seems largely prevented due to the weight of precedent in practice. In this paper, the view is taken that one of the issues is that there has not been a categorisation of underlying methodologies to provide a framework and terminology for better understanding of the relative strengths of alternative methods - thus leaving them breanded as alternative. In this paper therefore, we propose a typology and then expand on the detail of terminology applicable to the "alternative" branch.

There have been many attempts to categorize or generalize a collective term for that alternative family of scheduling methods which arise from handling repetitive production of units or physical locations. To avoid repetition, these are frequently encapsulated by reference to Harris and Ioannou (1998), however as this paper is specifically about the semantics of this family of methods, a selection of them are listed here:

- Harmonograms

- Line-of-balance

- Flowline or flow line

- Repetitive scheduling method

- Vertical production method

- Time-location matrix model

- Time space scheduling method

- Disturbance scheduling

- Horizontal and vertical logic scheduling for multistory projects 
- Horizontal and vertical scheduling

- Multiple repetitive construction process

- Representing construction

- Linear scheduling

- Time versus distance diagrams (T-D charts)

- Linear balance charts

- Velocity diagrams

Each term is descriptive, and in this they are extremely effective communicators. However, as each method differs, the result can be seen to be a plethora of terms.

Harris and Ioannou (1998) coined the term repetitive scheduling method (RSM) in an attempt to address this diversity by defining a collective term. Similarly, Russell and Wong (1993) proposed representing construction. However these attempts have not explored the underlying methodology or attempted to differentiate these methods from existing methodologies for scheduling projects, such as the critical path method (CPM). As a result, they remain descriptors rather than encapsulating a family of methods. In fact, to this day the earliest term line of balance remains the most commonly used collective term even though it actually does not describe many techniques at all well.

It is important to take the step of developing a typology of methodologies, because it is important to be able to differentiate each method's purpose and to correctly categorize the methods. In this way the relative strengths of a method may be highlighted and utilized, rather than each being seen as replaced by the next. Methods should seek to differentiate rather than to replace.

In this paper, two broad classifications are proposed for scheduling systems for construction. In the first instance, these are representative of the approach toward modeling construction activity. The two main categories are activity-based and location-based methodologies. In the process of preparing this categorization, a further basic breakdown of the two methodologies may be identified (refer Figure 1). For activity-based methods these are deterministic (CPM) and probabilistic (PERT). For location-based methods the division is into unit production and location production. All of the descriptors in the list above may divided between these latter two sub-categories.

Such a typology clarifies methods. In the real world of construction, where the critical path method dominates in practice, it is powerful to use these divisions to identify alternative strategies and to justify their use for specific purposes, or even to make the case that alternatives may be superior for planning and control of construction projects.

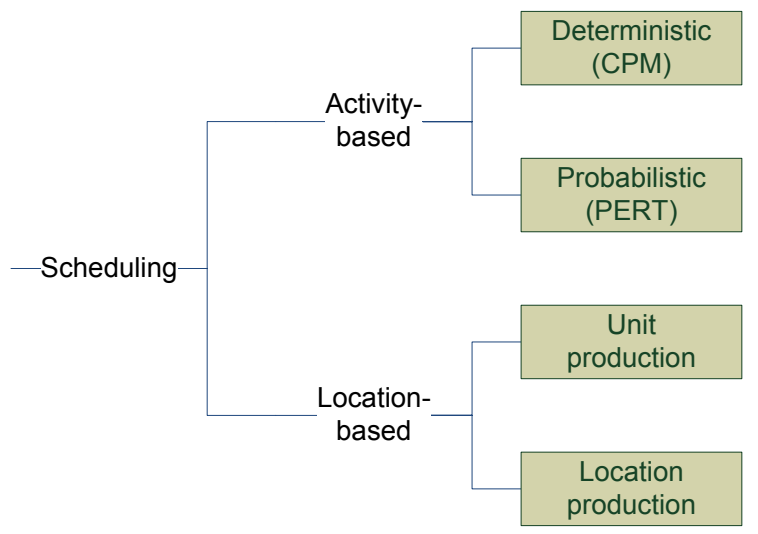

Figure 1: A typology for construction project scheduling methodologies

\section{A BROAD CATEGORISATION}

The first and most important division addresses the underlying epistemology of the scheduling methods. In this paper, this division is made by describing the approach and examining the assumptions of validity implicit in each methodology. By this method, two branches are found, termed here 1) activity-based and 2) location-based. 


\subsection{Activity-based methodology}

Activity-based scheduling is the current dominant scheduling technique and was first developed in the 1950s but owed much to the early work of Taylor and Gantt from the early 1900s. The company E.I. Du Pont de Nemours (DuPont) initiated research in 1956 which led to the seminal work of Kelley and Walker at the Univac Applications Research Centre in 1957. Papers by Kelley and Walker illustrated the model. They coined the term critical path method, the name selected... "because of the central position that critical activities in a project play in the method" (Kelley and Walker 1959).

The technique relies on the construction of a logical network of activities with two main divisions; deterministic (for example CPM) and probabilistic (for example PERT). Common to these is the underlying logical structure of the model. It is formed by a topological map of discrete activities joined by logical relationships. Each individual activity is considered free to move in time as long as it maintains the logical relationship with its predecessors and successors. Such a model suits very well any project where activities are completely discrete and have no structural correlation with other activities.

We have adopted the term activity-based to describe this methodology because the underlying epistemology is that a project may be modeled solely with individual packages of works (activities) and their logical relationships. The focus of the method is therefore on the activity as the container for project data, and the logical network is constructed by linking activities. The methodology is elegant and powerful, and has clearly enabled a rich environment for improving project performance.

There are two primary sub-divisions of activity-based methods, depending on whether the focus is on the calculation of a minimum duration for a complete network (a deterministic approach developed as the critical path method or CPM) as found in the current day application on most construction projects, or alternatively the focus being on the assessment of the likelihood (and risk) of meeting set deadlines (a probabilistic approach developed as the program evaluation and review technique or PERT) as found on major government strategic programs.

\section{$2.2 \quad$ Location-based methodology}

Location-based scheduling is an alternative methodology based on tracking the continuity of crews working on production tasks. These were originally graphical techniques, based on a technique designed as early as 1900 by Adamiecki (1931) and used as a construction management system as early as 1929 on such innovative projects as the Empire State Building, developed by the Goodyear Company in the 1940s and expanded by the US Navy in the 1950s. This suite of techniques found strong support in continuous general production systems (and is more typical in engineering construction) but has only found limited support in commercial construction, despite the large amount of research being undertaken in the 1960s and 1970s and indeed more recently.

This family of methods involve repetitive activities and for this Harris and Ioannou (1998) suggested the generic term repetitive scheduling method (RSM). However, the methods are more concerned with movement of resources through locations or places, and there really is no need for repetition. In fact, rather than the methodology focusing on repetition, it concentrates on tasks - which are aggregated activities over many production units or locations. The error with focusing on repetition is that this is actually an activity-based interpretation and the methodology should not define itself in terms of the dominant but inappropriate epistemology. Tasks are made of activities which "repeat" in multiple locations. Possibly this family of techniques should be called task-based methods, however this would be confusingly similar to activity-based techniques, thus the term location-based scheduling has been coined by the authors (Kenley 2004) as more appropriate and descriptive of the character of the methodology.

We have adopted the term location-based to describe this methodology because the underlying epistemology is that a project may be modeled by including individual packages of works (activities) into a connected whole entity called a task which represents the aggregation of activities in multiple locations. Topological models may then be constructed by linking tasks through a network of complex logical relationships. The focus of the method is therefore on the task moving through production units (locations) and the project data sits in both the task and the location. While in its basic form the methodology is largely graphical, modern methods have shown that it is possible to incorporate the full range of CPM logical relationships, both within and between tasks, and furthermore the presence of tasks and locations allows much more powerful relationships to be built. The methodology therefore has much greater power and flexibility for modeling construction projects.

There are two primary sub-divisions of location-based methods, depending on whether the focus is on the continuous completion of repetitive units (as found in repetitive factory production, or linear projects) as typified by line of balance method, or alternatively the focus being on the completion of variable physical locations (more typical in commercial construction) as typified by the flowline method. 


\subsubsection{Unit production}

With unit production, the emphasis is on the mathematics of production units per time period. The methods utilize a sloping line of commencement (of a repetitive activity or sub-network) and a line of completion (of the same activity or sub-network) on a chart of production versus time. This is typified by the line of balance representation as per Figure 2 .

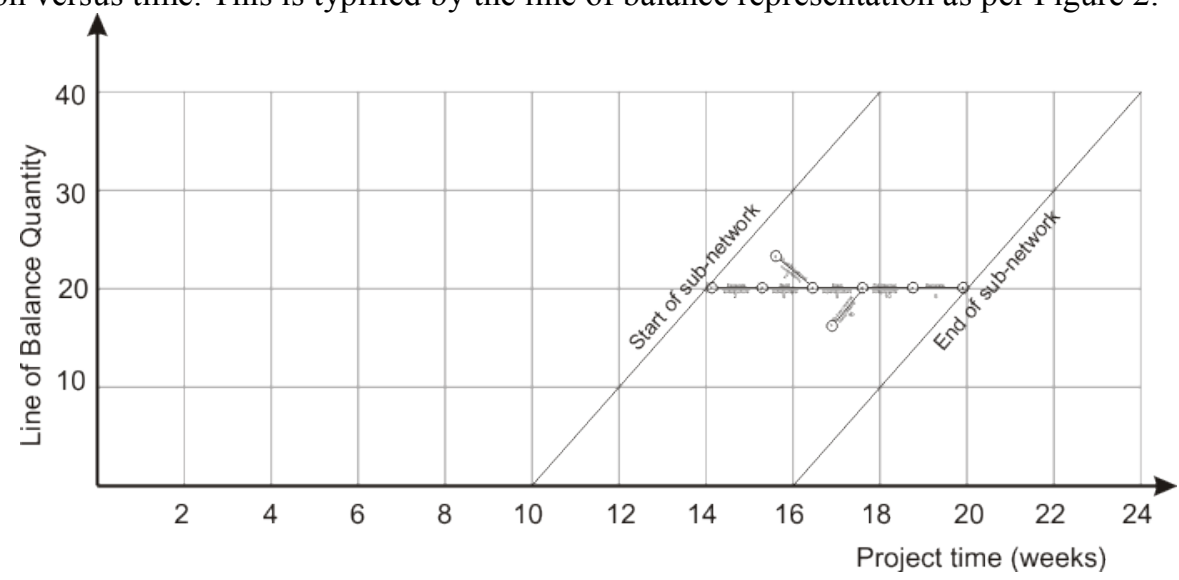

Figure 2: Line of balance showing line of commencement and line of completion (Kenley and Seppänen 2009 after Lumsden 1968)

Individual units do not matter with line of balance. The key is the rate of production of the line-of-balance quantity (the cumulative production). As the name suggests, the supporting management philosophy is to balance the production rates of differing production processes, as well as to use buffers to allow for variability (Figure 3).

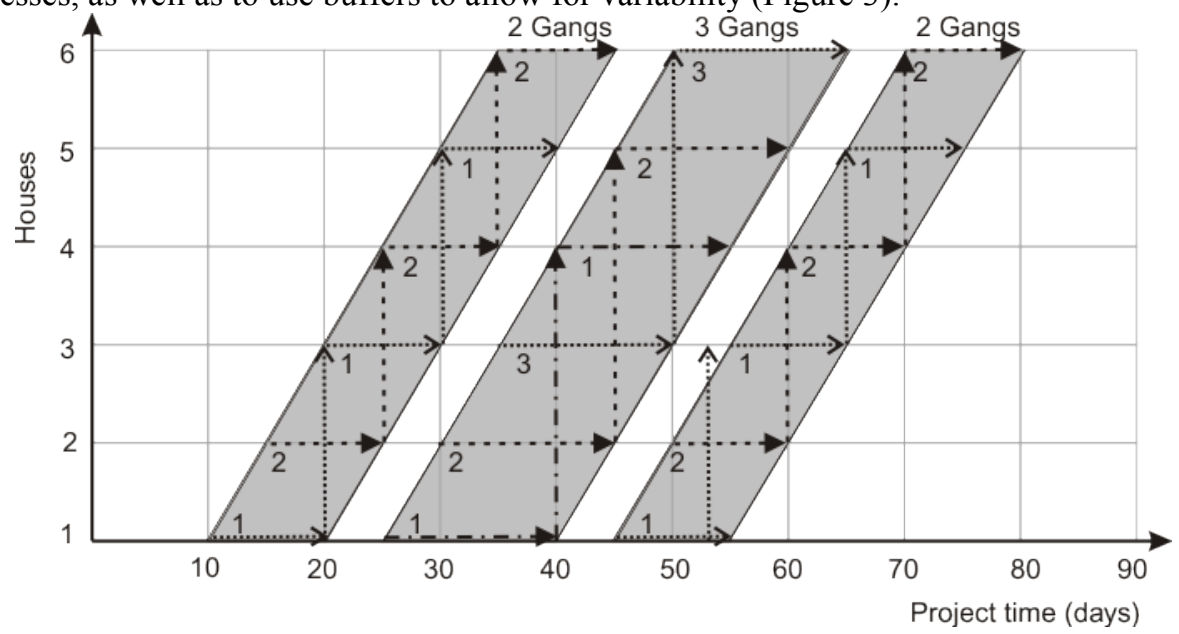

Figure 3: Balanced production of three tasks in line of balance (Kenley and Seppänen 2009 after NBA 1968)

\subsubsection{Location production}

With location production, the emphasis is on the execution of work within locations and the rate of sequential completion. This forms a single line for each task starting at the bottom of the location and finishing at the top of the location. Once one location is completed, the task continues into the second location. This is typified by the flowline representation as shown in Figure 4. 


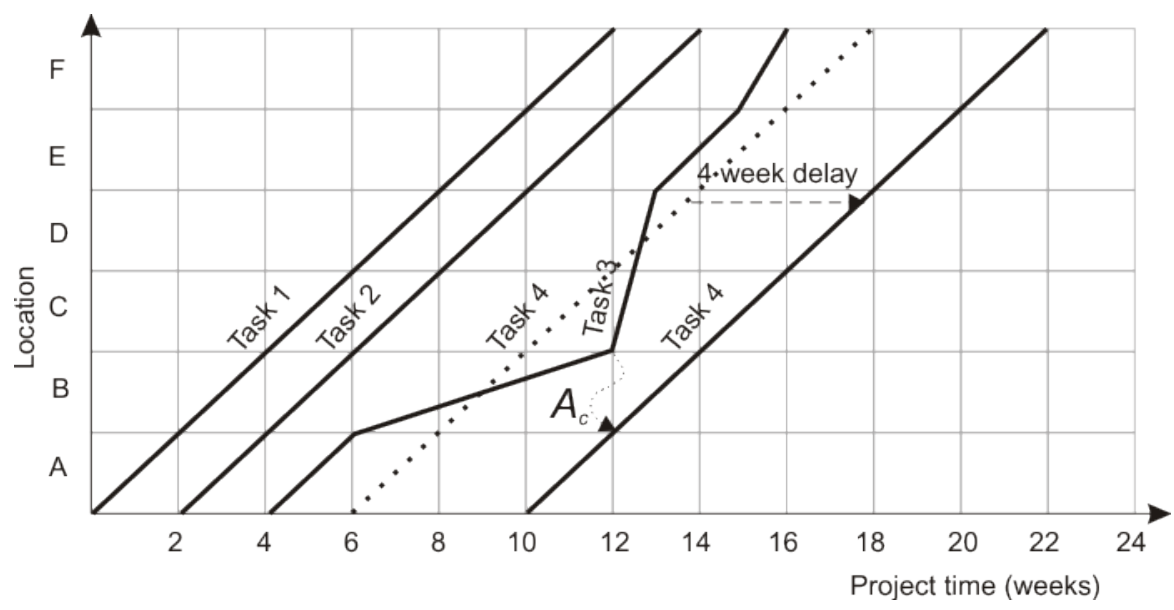

Figure 4: Flowline of four planned balanced tasks (showing effects of delay) (Kenley and Seppänen 2009)

The key issue is that individual locations very much matter with location production. The focus is on the rate of production of individual locations. As the name suggests, the supporting management philosophy is to flow resources for production processes through locations, as well as to use buffers to allow for variability.

Locations are more complex than production units, which can be dealt with numerically. Locations require the establishment of a hierarchical location breakdown structure (LBS) with the consequent benefit of taking on a physical reality as well as organizing the work on site. The LBS relates to a physical (or logical) breakdown of the project, such as shown in Figure 5, for which the LBS would as shown in Figure 6.

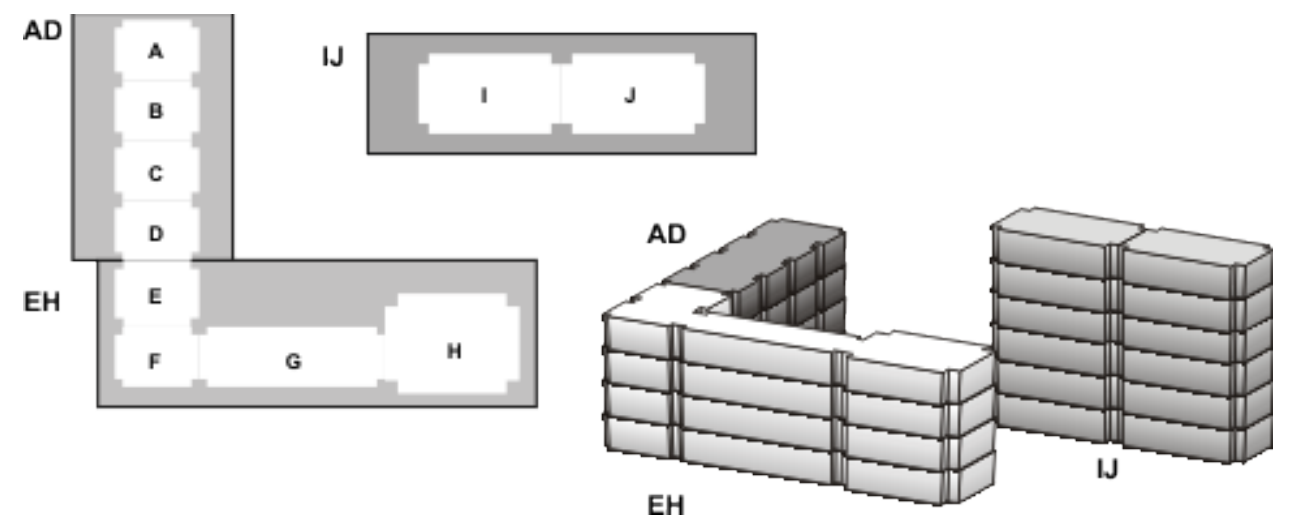

Figure 5: Typical project layout for preparing a LBS (Kenley and Seppanen, 2009)

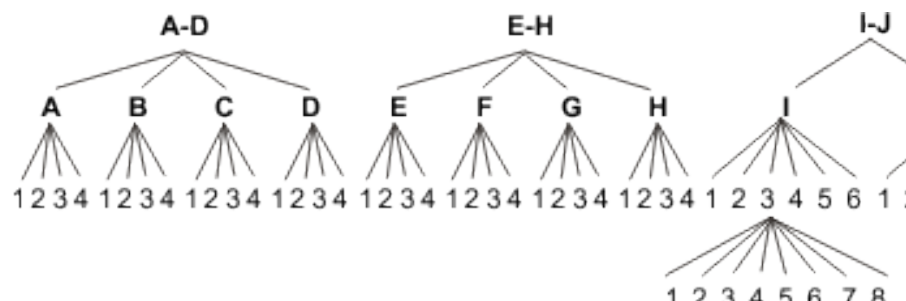

12345678

Section

Riser

Floor

Appartment

Figure 6: LBS for above typical project (Kenley and Seppänen 2009)

It should also be noted that as the production is of physical locations, there is no requirement for there to be repetition. Quantities are likely to vary (or be nonexistent) between locations, and required tasks may vary between locations. 
Kenley and Seppänen

\subsubsection{Choosing a term}

Ultimately, the term location-based can be used for both unit-production and location-production methodologies, because there is an overall picture of managing resources through continuous production rather than discrete activities. However it remains valuable to recognize the difference, because managing projects by line of balance does not force tasks to be treated as continuous (as they are represented is represented as overlapping) which has both advantages and disadvantages in planning and control of projects.

\section{LOCATION-BASED MANAGEMENT SYSTEM}

Locations are important in construction because building can rarely be seen as a continuous repetitive construction process, but rather as a series of physical locations in which work of variable type and quantity must be completed. It is the containerization of data into these locations to allows better planning and control to eventuate.

\subsection{Location-Unit of analysis}

Location as the unit of analysis is at the heart of the location based management system (LBMS). Location provides the container for all project data, and is used as the primary work division through a location breakdown structure (LBS) - rather than the more familiar work breakdown structure (WBS).

Location is the container for data which relates to the quantum of the project. The LBS is hierarchical so that a higher level location logically includes all the data of the lower level locations. Each of the location hierarchies have a different purpose. The highest level is used to optimize construction sequence, because the structures of such sections are independent of each other, therefore it is possible to start them in any sequence or to build them simultaneously. The middle levels are used to plan production flow of structure (and often reflect physical constraints). The lowest levels are used for planning detail and finishes. This allows data to be collected at different levels within the hierarchy. The location contains the following types of data:

- Building objects or components such as elements and sub-systems

Traditionally, building objects or components have been only available in drawings. Optimally, these should be documented in a 3D object oriented construction model. This may also include IFC (industry foundation classes) data for documentation data reuse.

- Planned and actual building component quantities

Quantities should preferably be measured directly from a 3D model. Measurement by a Quantity Surveyor or estimator is also possible, although manual measurement should be redundant with 3D model-based technology such as Vico Software's Vico Office. Variations in quantity which occur during construction should be able to be tracked.

- Building system production assemblies

In a construction system, the assembly of components is important, including the selected method of construction. Thus the assembly should include support components such as scaffolding and plant requirements such as cranage. These should be measured together with the measurement of materials quantities.

- Planned and actual material costs

Costs associated with planned and actual material quantities.

- Building system costs

Costs of all the components should be aggregated for each location and hierarchy level within the LBS. This process must be iterative, because labor costs should always be calculated based on the actual resource use reflected in the location-based plan.

\subsection{Task-Method of control}

The task is the method of control and is the container for data which relates to the production of the project, in particular, labor resources, time and cost. A task is the aggregation of all activities of the same type that repeat in multiple locations. Tasks have common resource requirements but quantities, crews and productivity will vary between locations. The task contains one or more quantity items, from all locations in the project, for work which can be aggregated into logical work. The decision to aggregate quantities comes down to deciding how the work will be carried out. If work is managed as one pack- 
age all the quantities that relate, rather than work which is clearly to be managed separately or at a different time, should be aggregated.

Quantities define the scope of the task and locations for the task (work may not exist in all locations). Some tasks necessarily become single-location activities.

Each task is defined at (and belongs to) a hierarchy level of the location breakdown structure. For example, the structure is raised one floor at a time, so the logical hierarchy level is Floor. Finishes are done one apartment at the time, so the logical hierarchy level is Apartment. The task contains the following types of data:

\section{- Standardized production data}

Standard consumption rates for ideal crews and resources. These provide a default for the resource planning process.

- Planned and actual resource requirements

Planned resource demands and actual resource consumption for the task.

\section{- Work crews}

The makeup and number of work crews for the planned and actual performance of the work. Work crews aggregate the resources for a given task and may themselves have properties.

- Logical constraints

The tasks have a logical relationship and sequence through the project. The layered CPM network logic belongs to tasks (see below).

- Pre-requisites for production

Any pre-requisites for production such as procurement, precedents, materials supply, etc. belong to the task as the method of control.

- Performance and forecasts

Past performance for the task is recorded and can be used to estimate future performance for that task.

\subsection{Full CPM functionality}

It is also important to realize that the location-based methodology does not exclude CPM. In fact, the logical relationships of the CPM methodology are applied as a superset of normal CPM relations. The location-based schedule requires that tasks can be linked using CPM logic. In fact, location-based logic requires that five layers of CPM logic be applied at the activity level (remembering that a task is made up of activities in individual locations). These are:

1 External logical relationships between activities within locations

2 External higher-level logical relationships between activities driven by different levels of accuracy

3 Internal logic between activities within tasks

4 Phased hybrid logic between tasks in related locations (location lags)

5 Standard CPM links between any tasks and different locations

Layered logic automatically generates CPM logic between two tasks by using locations-for example a task can be planned to happen before a successor in every location where they occur together. Layered logic is not hierarchical, instead all logics apply equally and the resultant forward and backward pass calculations are necessarily iterative and conditional in their function depending on the layer logic which applies in each situation. Standard CPM calculations can be used with the addition of heuristics which enable planning continuous work by pulling start dates of early locations.

In addition to the use of CPM logic lags and leads, location-based scheduling includes buffers. Buffers may be in either duration or location.

- Lags and leads: the required fixed duration of a logical connection between two activities or tasks.

- Buffers: The absorbable allowance for disturbance between adjacent tasks or locations is a component of the logical connection between two tasks but which may absorbed delay.

Buffers appear very similar to lags, except they are there to protect the schedule and are intended to absorb minor variations in production. 


\section{DISCUSSION}

There is much that is yet to be developed within the "alternative" planning, scheduling and controlling methods collectively referred to here as location-based methods. It is hoped that by clarifying the breakdown of methodologies based on epistemology underlying individual methods, will improve communication. Most importantly it will provide a basis for recognising the contribution which new techniques provide for practical construction management.

It really is time that industry moved beyond its reliance on a single method, CPM, and it explored the greater potential which new, location-based techniques offer.

\section{REFERENCES}

Adamiecki, K. 1931. "Czy Nauka Organizacji Przyczynia sie do Poglebiania Kryzysu i Bezrobocia? (L'organisation scientifique contribute-t-elle a approfondir la crise et augmenter le chomage?)". Przeglad Organizacji. 4(12), Warsaw.

Harris, R.B. and P.G. Ioannou, 1998. "Scheduling projects with repeating activities". Journal of Construction Engineering and Management 124(4): 269-278.

Kelley, J.E. and M.R. Walker, 1959. "Critical-Path Planning and Scheduling”. Proceedings of the Eastern Joint Computer Conference, 160-173.

Kenley, R. 2004. "Project micromanagement: Practical site planning and management of work flow". Proceedings of the 12th International conference of Lean Construction, Helsingør, Denmark, 194-205.

Kenley, R. and O. Seppänen, 2009. Location-based management for construction: Planning, scheduling and control. Spon Press, London and New York, (in press).

Lumsden, P. 1968. The Line-of-Balance method. Pergamon Press Limited: Industrial Training Division, London, 71pp.

NBA 1968. Programming House Building by Line of Balance, The National Building Agency, London: 24.

Russell, A.D. and W. Wong, 1993. "New Generation of Planning Structures". Journal of Construction Engineering and Management, 119(2): 196-214.

\section{AUTHOR BIOGRAPHIES}

RUSSELL KENLEY is Professor of Management at Swinburne University of Technology, Melbourne, Australia, and Visiting Professor of Construction at Unitec, Auckland, New Zealand. Holds a degree in Building (QS) and Ph.D. in Construction Economics and Management from The University of Melbourne. Hi research interests include financial management of construction, productivity and performance improvement focusing on location-based management, integrated project delivery focusing on BIM and strategic management of corporate real estate and public real estate. His email is $<$ rkenleyeswin.edu.au>

OLLI SEPPÄNEN is Vice President of Services Field Operations and Director of Production Control Solutions for Vico Software. He is the developer of new software tools to support location-based methods for construction, in particular Control (part of Vico Software virtual construction suite), a powerful tool for planning and controlling construction using flowline methods, as well as DynaRoad, a highly innovative tool for planning and controlling linear construction works. His email is <olli.seppanen@vicosoftware.com> 